University of Wollongong

Research Online

Australian Institute for Innovative Materials -

Papers

Australian Institute for Innovative Materials

$1-1-2013$

\title{
Nanosecond voltage pulses from dendritic flux avalanches in superconducting $\mathrm{NbN}$ films
}

P Mikheenko

University of Oslo

A J. Qviller

University of Oslo

J I. Vestgarden

University of Oslo

S Chaudhuri

University of Jyvaskyla

I J. Maasilta

University of Jyvaskyla

See next page for additional authors

Follow this and additional works at: https://ro.uow.edu.au/aiimpapers

Part of the Engineering Commons, and the Physical Sciences and Mathematics Commons

Research Online is the open access institutional repository for the University of Wollongong. For further information contact the UOW Library: research-pubs@uow.edu.au 


\title{
Nanosecond voltage pulses from dendritic flux avalanches in superconducting $\mathrm{NbN}$ films
}

\begin{abstract}
Combined voltage and magneto-optical study of magnetic flux flow in superconducting $\mathrm{NbN}$ films is reported. The nanosecond-scale voltage pulses appearing during thermomagnetic avalanches have been recorded in films partially coated by a metal layer. Simultaneous magneto-optical imaging and voltage measurements allowed the pulses to be associated with individual flux branches penetrating the superconductor below the metal coating. From detailed characteristics of pulse and flux branches, the electrical field in the superconductor is found to be in the range of $5-50 \mathrm{kV} / \mathrm{m}$, while the propagation speed of the avalanche during its final stage is found to be close to $5 \mathrm{~km} / \mathrm{s}$.
\end{abstract}

\section{Keywords}

superconducting, avalanches, flux, films, dendritic, nbn, pulses, voltage, nanosecond

\section{Disciplines}

Engineering | Physical Sciences and Mathematics

\section{Publication Details}

Mikheenko, P., Qviller, A. J., Vestgarden, J. I., Chaudhuri, S., Maasilta, I. J., Galperin, Y. M. \& Johansen, T. H. (2013). Nanosecond voltage pulses from dendritic flux avalanches in superconducting NbN films. Applied Physics Letters, 102 (2), 022601-1-022601-3.

\section{Authors}

P Mikheenko, A J. Qviller, J I. Vestgarden, S Chaudhuri, I J. Maasilta, Y M. Galperin, and Tom H. Johansen 


\section{AIP Appiled Physics \\ Letters}

\section{Nanosecond voltage pulses from dendritic flux avalanches in superconducting $\mathrm{NbN}$ films}

P. Mikheenko, A. J. Qviller, J. I. Vestgården, S. Chaudhuri, I. J. Maasilta et al.

Citation: Appl. Phys. Lett. 102, 022601 (2013); doi: 10.1063/1.4775693

View online: http://dx.doi.org/10.1063/1.4775693

View Table of Contents: http://apl.aip.org/resource/1/APPLAB/v102/i2

Published by the American Institute of Physics.

\section{Related Articles}

Planar defects, dislocations, and coherently scattering-size in $\mathrm{GdBa} 2 \mathrm{Cu} 3 \mathrm{O} 7-\mathrm{x}$ high-Tc thin films determined by high resolution X-ray diffraction

J. Appl. Phys. 113, 033903 (2013)

Magneto-elastic behaviour of thin type-II superconducting strip with field-dependent critical current J. Appl. Phys. 113, 023901 (2013)

High-temperature superconducting multi-band radio-frequency metamaterial atoms

Appl. Phys. Lett. 102, 013503 (2013)

Reversible superconductivity in electrochromic indium-tin oxide films

Appl. Phys. Lett. 101, 252603 (2012)

Homogeneous superconducting phase in TiN film: A complex impedance study

Appl. Phys. Lett. 101, 252601 (2012)

\section{Additional information on Appl. Phys. Lett.}

Journal Homepage: http://apl.aip.org/

Journal Information: http://apl.aip.org/about/about_the_journal

Top downloads: http://apl.aip.org/features/most_downloaded

Information for Authors: http://apl.aip.org/authors

\section{ADVERTISEMENT}

\section{AIP Applied Physics Letters}

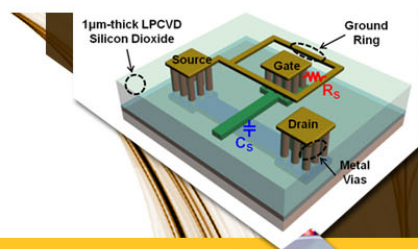

\section{SURFACES AND} INTERFACES

Focusing on physical, chemical, biological structural, optical, magnetic and electrical properties of surfaces and interfaces, and more..

\section{EXPLORE WHAT'S NEW IN APL}

SUBMIT YOUR PAPER NOW!
ENERCY CONVERSION AND STORACE 


\title{
Nanosecond voltage pulses from dendritic flux avalanches in superconducting $\mathrm{NbN}$ films
}

\author{
P. Mikheenko, ${ }^{1, a)}$ A. J. Qviller, ${ }^{1}$ J. I. Vestgården, ${ }^{1}$ S. Chaudhuri, ${ }^{2}$ I. J. Maasilta, ${ }^{2}$ \\ Y. M. Galperin, ${ }^{1,3,4}$ and T. H. Johansen ${ }^{1,3,5}$ \\ ${ }^{1}$ Department of Physics, University of Oslo, P.O. Box 1048, Blindern, 0316 Oslo, Norway \\ ${ }^{2}$ Nanoscience Center, Department of Physics, P.O. Box 35, University of Jyväskylä, FIN-40014 Jyväskylä, \\ Finland \\ ${ }^{3}$ Centre for Advanced Study at the Norwegian Academy of Science and Letters, 0271 Oslo, Norway \\ ${ }^{4}$ Ioffe Physical Technical Institute, 26 Polytekhnicheskaya, St. Petersburg 194021, Russia \\ ${ }^{5}$ Institute for Superconducting \& Electronic Materials, University of Wollongong, NSW 2522, Australia
}

(Received 3 October 2012; accepted 26 December 2012; published online 15 January 2013)

\begin{abstract}
Combined voltage and magneto-optical study of magnetic flux flow in superconducting NbN films is reported. The nanosecond-scale voltage pulses appearing during thermomagnetic avalanches have been recorded in films partially coated by a metal layer. Simultaneous magneto-optical imaging and voltage measurements allowed the pulses to be associated with individual flux branches penetrating the superconductor below the metal coating. From detailed characteristics of pulse and flux branches, the electrical field in the superconductor is found to be in the range of $5-50 \mathrm{kV} / \mathrm{m}$, while the propagation speed of the avalanche during its final stage is found to be close to $5 \mathrm{~km} / \mathrm{s}$. C 2013 American Institute of Physics. [http://dx.doi.org/10.1063/1.4775693]
\end{abstract}

Superconductivity is going to play an important role in the development of an economy based on renewable energy resources. ${ }^{1}$ To achieve the highest performance, superconducting materials are likely to be used at the limit of their current carrying capability. This, however, may lead to thermomagnetic breakdown, an instability which is well-known but difficult to prevent in most superconducting materials. ${ }^{2,3}$ It can take place in bulk samples, wires, and tapes, but in thin films subjected to a perpendicular magnetic field, the instability takes a most spectacular form of dendritic avalanches propagating at ultra-fast speeds. The phenomenon has been reported to occur in a large number of materials important for practical applications, such as $\mathrm{Nb},{ }^{4} \mathrm{NbN},{ }^{5}$ $\mathrm{Nb}_{3} \mathrm{Sn},{ }^{6}$ and $\mathrm{MgB}_{2}{ }^{7}$ The avalanches are expected to generate strong electromagnetic noise and even destroy superconducting equipment. ${ }^{8}$

Whereas flux structures frozen in superconducting films after such events are routinely observed by magneto-optical imaging (MOI), ${ }^{4-7}$ experiments providing insight to the time-resolved behavior of these ultrafast avalanches are extremely few. Previous work on films of $\mathrm{YBa}_{2} \mathrm{Cu}_{3} \mathrm{O}_{\mathrm{x}}$ has combined MOI and a femtosecond pulsed laser technique, where an avalanche was triggered by a laser-generated hot spot, and a few nanoseconds later laser light was used to record a snapshot of the evolving flux distribution. ${ }^{9}$ From such measurements, it was found that at the initial stage the flux branches grow at speeds near a hundred kilometers per second. Using that method, however, little information was obtained about the dynamic electrical field generated during avalanche events. In this work, we present fast voltage measurements in combination with MOI, allowing determination of the transient electrical voltage, as well as electrical and magnetic fields associated with the propagation of dendritic flux avalanches.

${ }^{a)}$ E-mail: pavlo.mikheenko@fys.uio.no.
Figure 1 illustrates the sample configuration, where a $170 \mathrm{~nm}$ thick film of superconducting $\mathrm{NbN}$ is covered on the left half by a $900 \mathrm{~nm}$ thick $\mathrm{Cu}$ layer. The $\mathrm{NbN}$ was grown on $\mathrm{MgO}(001)$ single crystals using a pulsed laser deposition technique. ${ }^{10}$ Then, using electron beam lithography (EBL) and reactive ion etching with $\mathrm{CF}_{4}+\mathrm{O}_{2}$, a rectangular $\mathrm{NbN}$ area was patterned on the chip. In the final step, the copper layer was deposited on one-half of the $\mathrm{NbN}$ area using overlay EBL and electron-beam evaporation. Finally, two electrical contacts were placed near the lower corners of the sample area, which measures $3.0 \times 1.5 \mathrm{~mm}^{2}$. Previous MOI work has shown that corner regions almost never experience flux avalanches, ${ }^{11}$ and are thus optimal locations for placing electrical contacts. As a voltmeter, an oscilloscope Tektronix TDS 210 with nanosecond resolution and triggering mode was used. It was set to record voltage with sampling intervals

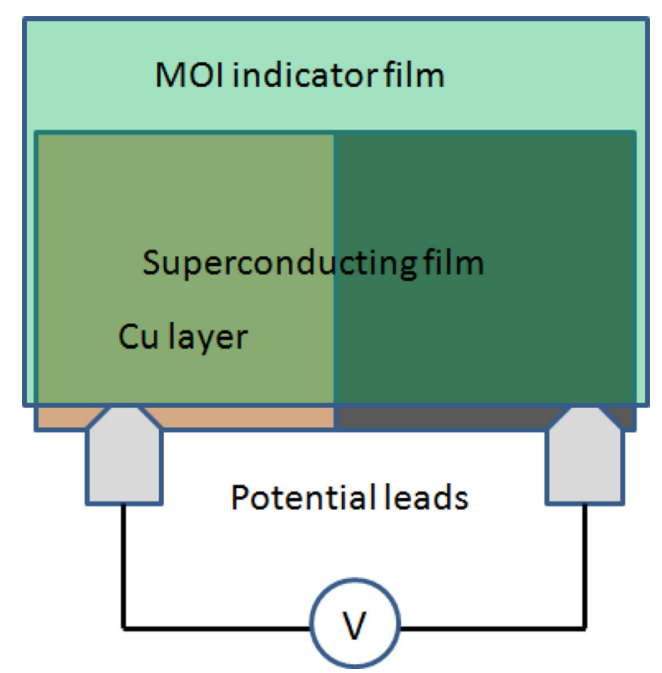

FIG. 1. Experimental configuration for simultaneous magneto-optical imaging and voltage measurements. 
down to $0.1 \mathrm{~ns}$, and was triggered by the pulse itself when instantaneous voltage was exceeding a threshold value, which was varied in experiments from 1 to $100 \mathrm{mV}$. When triggered, the voltmeter also stored previously measured data in a preset time interval. Before attaching the potential leads, the sample was thoroughly investigated by MOI, ${ }^{12,13}$ where a ferrite garnet indicator film covered the entire sample area.

Figure 2(a) shows the flux distribution when the sample was initially zero-field-cooled to $7.5 \mathrm{~K}$, and then exposed to a perpendicular magnetic field of $12.8 \mathrm{mT}$. The image brightness represents the magnitude of the local flux density, and the pattern shows that the film is macroscopically uniform. The flux has penetrated approximately $0.2 \mathrm{~mm}$ into the sample from all edges, which corresponds to having a critical current density of $j_{\mathrm{c}}=2.3 \times 10^{11} \mathrm{~A} / \mathrm{m}^{2}$. The image shows no indications that the $\mathrm{Cu}$ layer influences the superconducting properties of the $\mathrm{NbN}$ film in any significant way. The ramification of the flux front is caused by micro-defects and edge roughness. The large dendritic structure rooted at the lower edge of the film shows the flux pattern created by a typical avalanche event. The vertical dashed line marks the bound-
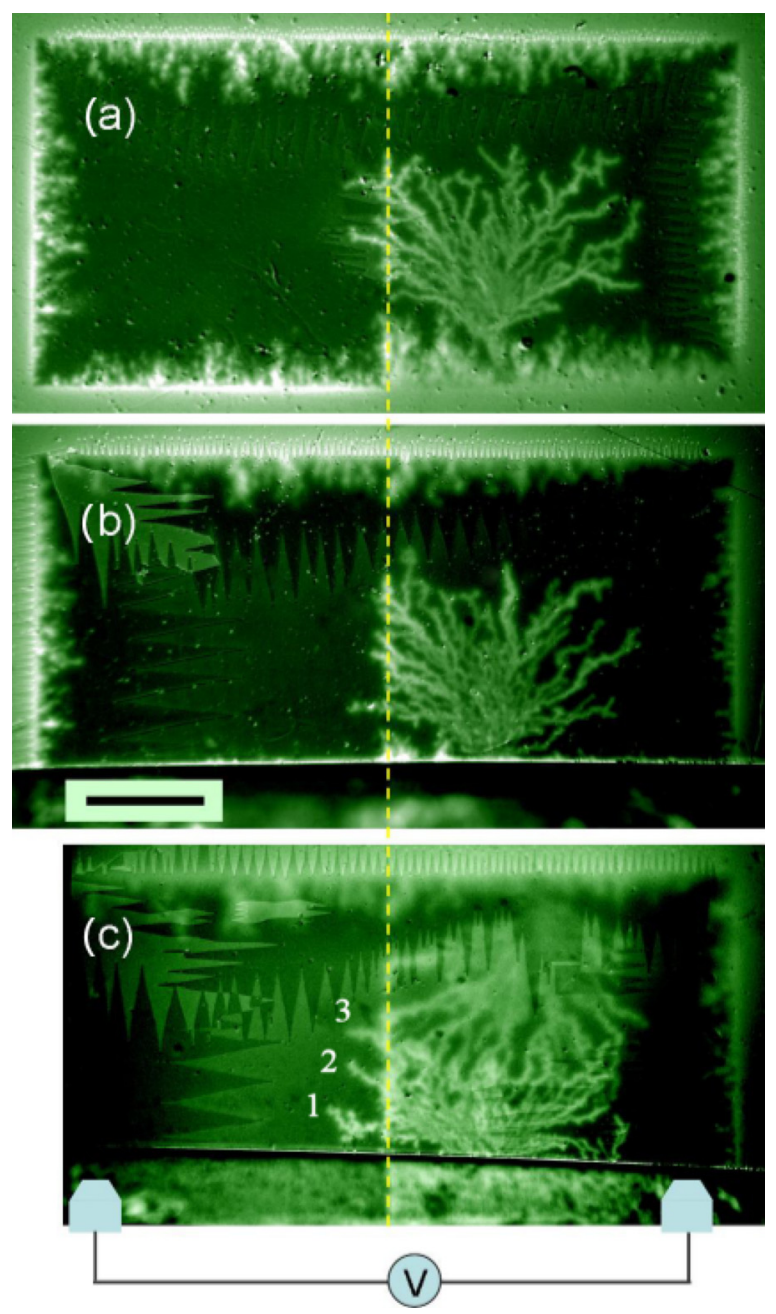

FIG. 2. Dendritic avalanches in the NbN film before (a) and after (b), (c) attaching potential leads. The boundary between the metal cover on the left and the bare $\mathrm{NbN}$ film on the right is indicated by the dashed line. The length of the black scale bar in (b) is $0.5 \mathrm{~mm}$. The horizontal line in the lower region of (b) and (c) is the edge of the indicator film. The bright features below this line in (c) are artefacts of light reflections from the metal surface, and are independent of the applied magnetic field. ary between the $\mathrm{Cu}$-coated (left) and bare (right) parts of the superconducting film, and one sees that a few flux branches have penetrated also into the $\mathrm{Cu}$-covered area.

It is already known that a normal metal layer adjacent to a superconducting film tends to suppress the avalanche activity due to electromagnetic braking, ${ }^{14-16}$ an effect experienced also in the present work, where we never observed avalanches starting from an edge covered with metal film. However, the $\mathrm{Cu}$ layer on the present $\mathrm{NbN}$ sample is sufficiently thin to allow branches to partly enter under the $\mathrm{Cu}$ electrode.

With the triggering option described above, voltmeter never registers any pulse when dendritic flux avalanches are outside of $\mathrm{Cu}$-layer, even if they come very close to this layer. In contrast, when avalanches penetrate into the $\mathrm{Cu}$ layer, voltmeter registers a strong pulse of the amplitude of several hundred $\mathrm{mV}$ that lasts few tens of nanoseconds. It means that we are only capable of measuring the induced voltage in the $\mathrm{Cu}$-layer. This is reasonable to expect given that electric field in the superconductor is efficiently screened by supercurrents, except inside the normal cores of the propagating branches. In its turn, the fact that there is a difference between potentials of the $\mathrm{Cu}$-layer and the superconductor means that there is either a significant interface resistance or a capacitive layer between them. The existence of such a layer is to be expected since the $\mathrm{NbN}$ film was exposed to air before the $\mathrm{Cu}$-layer was deposited. In this respect, the system is similar to that described in Ref. 16, where dendritic avalanches were suppressed by electromagnetic braking with clear separation between the metal and the superconductor. In that work, the voltage was not measured, but the presence of electromagnetic braking is a clear indication of the existence of a significant voltage induced in the metal by the fast dendritic flux avalanches in the superconductor.

Shown in Fig. 3(a) is the voltage signal generated by the particular avalanche seen in the magneto-optical image in Fig. 2(b). With the potential leads attached, the indicator film is here slightly shifted to get clear from the contacts. The measured voltage, which is an integral signal from all branches entering into the $\mathrm{Cu}$-coated area, forms a welldefined pulse with a half-width of $40 \mathrm{~ns}$, and a peak value of $0.37 \mathrm{~V}$. From the pulse shape, we conclude that in this avalanche event, all the four easily identifiable flux branch segments arrived under the electrode within an interval of approximately $40 \mathrm{~ns}$.

Figure 3(b) shows the voltage signal from another experiment on the same sample as seen in Fig. 2(c). Here, the first avalanche, starting from the upper edge, did not enter under the $\mathrm{Cu}$-electrode and also did not trigger the voltmeter. However, the second avalanche rooted at the lower edge did result in the shown voltage signal, which clearly reveals a fine structure caused by several flux branches entering the $\mathrm{Cu}$-electrode at different times. When decomposing the signal into separate pulses, each individual branch contributes by a peak of half-width $30 \mathrm{~ns}$, but of different magnitudes. The numbers assigned to the branches in Fig. 2(b) and to the voltage peaks in Fig. 3(b) indicate a suggested correspondence between the different pulses and respective flux branches, assuming that the voltage amplitude depends on how far from the electrode connection line the flux motion takes place. ${ }^{17,18}$ 

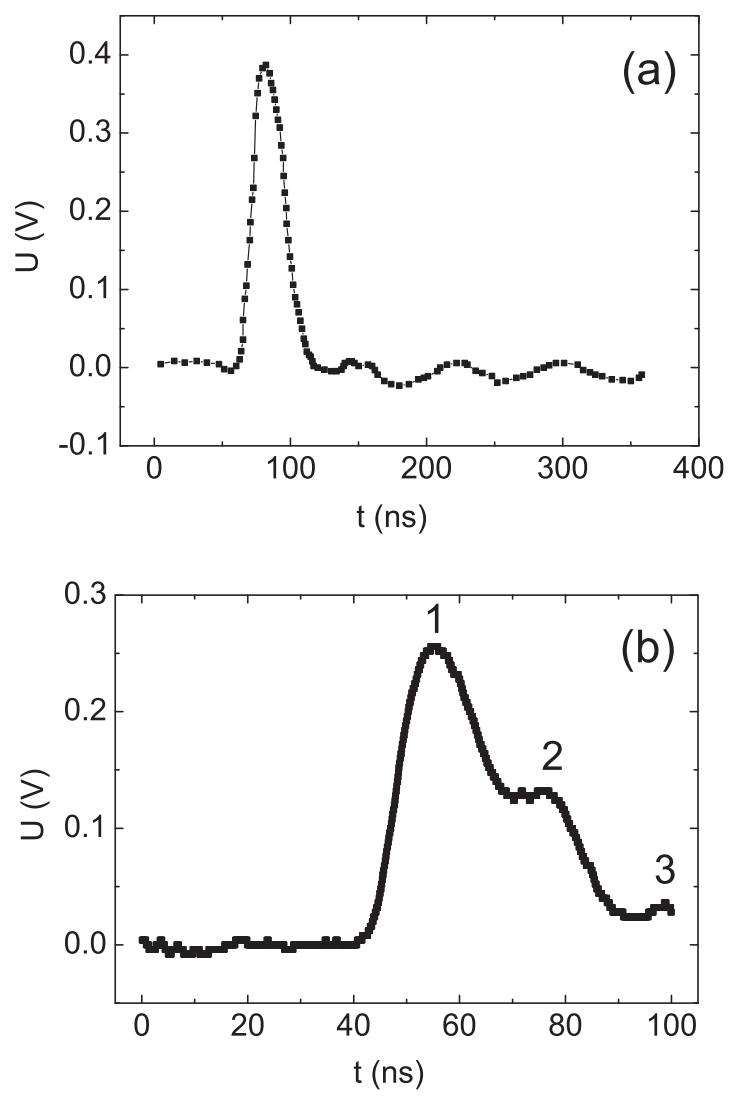

FIG. 3. (a) Voltage pulse generated by dendritic avalanche in Fig. 2(b) at temperature of $3.8 \mathrm{~K}$ and applied magnetic field of $4.25 \mathrm{mT}$. The measured pulse is an integral signal from all branches entering into the $\mathrm{Cu}$-coated area. (b) Voltage pulse generated by dendritic avalanche shown in Fig. 2(c) at temperature of $4.5 \mathrm{~K}$ and applied magnetic field of $2.25 \mathrm{mT}$. The numbers in Figs. 2(c) and (b) indicate a suggested correspondence between the different pulses and respective flux branches.

The simultaneous recording of voltage pulses and magneto-optical images allows interesting estimates to be made. From the length of the branch segments penetrating under the metal coating, and the duration of the voltage pulses, the avalanche propagation speed can be determined. Using the results shown in Fig. 2(c), where individual branches are resolved, we find an average speed of $5 \mathrm{~km} / \mathrm{s}$, using a branch length of $0.15 \mathrm{~mm}$ formed during a time interval (equal to the pulse halfwidth) of $30 \mathrm{~ns}$. This speed is 3-4 times less than that reported by Bolz et al. ${ }^{9}$ for the late stage of dendritic avalanches in $\mathrm{YBa}_{2} \mathrm{Cu}_{3} \mathrm{O}_{\mathrm{x}}$. At $10 \mathrm{~K}$, they found a speed, which in the very early stages of an avalanche has values near $150 \mathrm{~km} / \mathrm{s}$, and then decreasing to $18 \mathrm{~km} / \mathrm{s}$ at the later stages. In our experiment, the measured late-stage speed is lower, which is to be expected since the branch propagation gets further slowed down by the presence of $\mathrm{Cu}$-layer.

In the present measurements, we find that the width of the flux branches below the electrode is near $20 \mu \mathrm{m}$, an observation which can be used to estimate the electrical field. Since the voltage close to the branch is as high as $1 \mathrm{~V}$, it follows that the electrical field can be as high as $50 \mathrm{kV} / \mathrm{m}$. The result is in good agreement with recent simulations of the dynamics of the thermomagnetic instability by Vestgården et al. ${ }^{19}$ where both high speed of avalanche propagation and large generated electrical field have been found.
In summary, we have reported a relatively simple experimental technique, which allows imaging of dendritic avalanches in combination with measurements of the time dependence of the induced electrical voltage, on nanosecond time scales. These measurements have been used to estimate the speed of the avalanche propagation and the value of the generated electrical field. We have found that an average speed of propagation during the final stage of an avalanche is $5 \mathrm{~km} / \mathrm{s}$. We suggest that the ultrafast dynamics of an avalanche is linked to the high electrical field generated during its propagation, which in our experiment reaches values as high as $50 \mathrm{kV} / \mathrm{m}$.

From an applied perspective, the generation of the described voltage signals, though being a hallmark of flux motion in a normal metal film, is of major concern for superconducting and hybrid devices. While it was shown previously $^{14,16}$ that coating a superconductor film with a metal layer strongly suppresses the dendritic instability, we demonstrate here that when flux dendrites actually penetrate into a coated region, they may have a serious impact on the device performance. Therefore, thin superconducting devices experiencing transverse magnetic fields should be carefully designed to be robust against electrical noise of the type reported in this work.

The financial support of The Research Council of Norway was gratefully acknowledged. Research at the University of Jyväskylä was supported by the Academy of Finland within Project No. 128532.

${ }^{1}$ P. Mikheenko, J. Phys.: Conf. Ser. 286, 012014 (2011).

${ }^{2}$ R. G. Mints and A. L. Rakhmanov, Rev. Mod. Phys. 53, 551 (1981)

${ }^{3}$ D. V. Denisov, A. L. Rakhmanov, D. V. Shantsev, Y. M. Galperin, and T. H. Johansen, Phys. Rev. B 73, 014512 (2006).

${ }^{4}$ C. A. Durán, P. L. Gammel, R. E. Miller, and D. J. Bishop, Phys. Rev. B 52, 75 (1995).

${ }^{5}$ I. A. Rudnev, D. V. Shantsev, T. H. Johansen, and A. E. Primenko, Appl. Phys. Lett. 87, 042502 (2005).

${ }^{6}$ I. A. Rudnev, S. V. Antonenko, D. V. Shantsev, T. H. Johansen, and A. E. Primenko, Cryogenics 43, 663 (2003).

${ }^{7}$ T. H. Johansen, M. Baziljevich, D. V. Shantsev, P. E. Goa, Y. M. Galperin, W. N. Kang, H. J. Kim, E. M. Choi, M.-S. Kim, and S. I. Lee, Europhys. Lett. 59, 599 (2002).

${ }^{8}$ P. Brüll, D. Kirchgässner, P. Leiderer, P. Berberich, and H. Kinder, Ann. Phys. 504, 243 (1992).

${ }^{9}$ U. Bolz, B. Biehler, D. Schmidt, B.-U. Runge, and P. Leiderer, Europhys. Lett. 64, 517 (2003).

${ }^{10}$ S. Chaudhuri, M. R. Nevala, T. Hakkarainen, T. Niemi, and I. J. Maasilta, IEEE Trans. Appl. Supercond. 21, 143 (2011).

${ }^{11}$ J. I. Vestgården, Y. M. Galperin, and T. H. Johansen, Physica C 479, 92 (2012).

${ }^{12}$ P. E. Goa, H. Hauglin, A. A. F. Olsen, M. Baziljevich, and T. H. Johansen, Rev. Sci. Instrum. 74, 141-146 (2003).

${ }^{13}$ L. E. Helseth, R. W. Hansen, E. I. Il'yashenko, M. Baziljevich, and T. H. Johansen, Phys. Rev. B 64, 174406 (2001).

${ }^{14}$ M. Baziljevich, A. V. Bobyl, D. V. Shantsev, E. Altshuler, T. H. Johansen, and S. I. Lee, Physica C 369, 93 (2002).

${ }^{15}$ E. M. Choi, V. V. Yurchenko, T. H. Johansen, H.-S. Lee, J. Y. Lee, W. N. Kang, and S.-I. Lee, Supercond. Sci. Technol. 22, 015011 (2009).

${ }^{16}$ F. Colauto, E. Choi, J. Y. Lee, S. I. Lee, E. J. Patiño, M. G. Blamire, T. H. Johansen, and W. A. Ortiz, Appl. Phys. Lett. 96, 092512 (2010).

${ }^{17}$ R. P. Huebener, Magnetic Flux Structures in Superconductors (SpringerVerlag, Berlin, Heidelberg, 2001).

${ }^{18}$ J. R. Clem, Phys. Rev. B 1, 2140 (1970).

${ }^{19}$ J. I. Vestgården, D. V. Shantsev, Y. M. Galperin, and T. H. Johansen, Phys. Rev. B 84, 054537 (2011). 